\title{
Motor Abilities and the Motor Profile in Individuals with Williams Syndrome
}

\author{
Leighanne A. Mayall ${ }^{1}$ (D) - Hana D'Souza ${ }^{2}$ - Elisabeth L. Hill ${ }^{3} \cdot$ Annette Karmiloff-Smith $^{2} \cdot$ Andrew Tolmie $^{1}$. \\ Emily K. Farran ${ }^{4}$
}

Published online: 8 July 2020

(C) The Author(s) 2020

\begin{abstract}
Objectives Motor difficulties are present across a range of neurodevelopmental disorders, impacting on the development of other domains and on overall quality of life. One population that shows difficulties with their motor abilities is composed of individuals with Williams syndrome (WS). The purposes of the current study were to investigate the motor profile of individuals with WS and to investigate the relationships between physical activity and motor performance in this group.

Methods The motor performance of 36 individuals with WS was measured using the Bruininks-Oseretsky Test of Motor Proficiency, second edition (BOT2-SF) short form. Physical activity was also measured using our novel questionnaire. Performance on both measures was compared with that of typically developing (TD) children aged 4 to 7 years $(N=40)$.

Results Results indicate that the individuals with WS (aged 12 to 50 years) performed at the level of TD 4- to 5-year olds with respect to overall motor ability. On examination of the motor profile, a relative strength in upper limb control and a relative weakness in balance were identified for this group. While a correlation was found between motor ability and the amount of physical activity that participants engaged in on a weekly basis in the TD group, no such relationship was found in the WS group. Conclusions The motor problems that individuals with WS show in childhood persist into older childhood and adulthood, and akin to the WS cognitive profile, there are relative strengths and weaknesses in the WS motor profile. The lack of correlation between physical activity and motor ability in the WS group may be due to the lack of opportunity to access age- and abilityappropriate activities.
\end{abstract}

Keywords Williams syndrome $\cdot$ Motor $\cdot$ Physical activity $\cdot$ Neurodevelopmental disorders $\cdot$ Learning difficulties

Successful motor performance is one of the most basic and important skills for a typically developing child to master (Shaffer and Kipp 2010). From birth, typically developing infants begin their cognitive development by means of

Leighanne A. Mayall

leighanne.mayall.14@ucl.ac.uk

Emily K. Farran

e.farran@surrey.ac.uk

1 Psychology and Human Development, UCL Institute of Education, University College London, 25 Woburn Square, London WC1H 0AA, UK

2 University of Cambridge, Cambridge CB2 1TN, UK

3 Goldsmiths, University of London, Bloomsbury, London WC1E 7HU, UK

4 School of Psychology, University of Surrey, Guildford, Surrey GU2 7XH, UK engaging with their environment through motor acts. For example, they react to interesting stimuli by orientating their head (Bertenthal 1996). These simple movements, over time, evolve into more complex motor behaviours, which in turn aid the acquisition of new motor skills, such as sitting unsupported, standing, and eventually, walking. The acquisition of new motor abilities further enables new learning opportunities beyond the motor domain, such as visuospatial abilities (Bushnell and Boudreau 1993), daily living ability (Watkinson et al. 2001), language development (Iverson 2010), and spatial abilities (e.g. Clearfield 2004; Frick and Möhring 2013; Schwarzer et al. 2013). This suggests that there is also an association between motor abilities and cognitive abilities. Furthermore, social skills (Cummins et al. 2005; Green et al. 2006; Wagner et al. 2012) and emotional regulation accuracy (Chen and Bargh 1999; Coombes et al. 2006; Coombes et al. 2008; Coombes et al. 2005; Gross et al. 2012; Rotteveel and Phaf 2004) have been shown to be associated with motor ability. 
One dominant theory of motor development and motor learning is the dynamical systems theory (DST; Thelen and Smith 1998). The DST framework is split into internal factors, such as cognition, genetics, brain structure and function, and the body (e.g. physical deformities, hormones); and external factors, such as available space, task demands, distractions, and any other influence external to the individual. Under this theory, movement is thought to be the result of the interaction between multiple cognitive and internal systems, the environment, and the task at hand, all of which work together to help shape the performance of motor actions. The advantage of the DST model is that it can afford flexibility of motor actions in different environmental conditions, for example, both familiar and unfamiliar environments. Given the complexity of the theory, it is an appropriate framework within which to investigate how movement develops and changes and how learning occurs in relation to motor acts.

Given the importance of successful motor development, it is likely that motor impairments have wide-reaching consequences to many aspects of physical, cognitive, and social development. One population who present with motor deficits are individuals with Williams syndrome (WS). Individuals with WS have a partial deletion of chromosome 7 of around 25 to 28 genes (see Lenhoff et al. 1997). These individuals present with atypicalities in brain structure and function, which are associated with mild to moderate learning difficulties (Bellugi et al. 1994; Wang and Bellugi 1994), and are likely to impact their psychomotor development (i.e. the relationship between physical movement and cognitive activity) (Hocking et al. 2008).

A number of factors have been proposed to contribute to motor difficulties in WS. Schneider et al. (2012) investigated the role of GTF2IRD1, which is deleted in WS, using mice knockout models. They discovered that the mice who had the GTF2IRD1 gene knocked out showed decreased spontaneous motor activity, deficits in motor coordination, gait abnormalities, and reduced strength, which suggest a possible role of GTF2IRD1 in the motor deficit observed in WS.

From the few neuroimaging studies involving individuals with WS, there is evidence to suggest that there are differences in the size of several brain areas in this population, including the cerebellum and basal ganglia, both of which are associated with the control and execution of motor acts (Jernigan and Bellugi 1990; Jernigan et al. 1993; Jones et al. 2002; Reiss et al. 2000). Data examining the total volume of the cerebellum in WS has been inconsistent, with some authors finding an increase in cerebellar volume relative to chronological agematched controls and overall brain size in WS (Jones et al. 2002; Reiss et al. 2000), and others finding no differences in volume in comparison with typical controls (Chiang et al. 2007; Jernigan et al. 1993). Overall, these studies suggest that the absolute volume of the cerebellum in WS is comparable to healthy chronological age-matched controls, but that there is a slight increase in the relative volume of this structure, when the overall volume reduction of the cerebrum is considered. Furthermore, it has been reported by Jernigan et al. (1993) and Reiss et al. (2000) that the basal ganglia of individuals with WS are reduced in size and volume compared with typically developing chronological age-matched participants. The basal ganglia play a key role in the control of movement (Monchi et al. 2006), and so it is possible that this reduction in size could be detrimental to individuals in this population with reference to carrying out motor acts successfully.

It has also been suggested by Atkinson et al. (1997) that the reported difficulties in the dorsal visual stream (which runs from the occipital lobe to the parietal lobe) of individuals with WS affect not only their spatial skills, but also their motor actions, such as walking over uneven surfaces (Withers 1996). Furthermore, behavioural research that has used tasks to investigate the role of the posterior parietal lobe in individuals with WS has shown impairments in visually guided actions when compared with typically developing mental agematched controls (Atkinson et al. 1997), suggesting atypicality in the dorsal stream functions related to controlling movement. To summarise, the above studies imply that there are both structural and functional differences in several brain areas that are thought to be associated with the control of motor acts in WS (although no studies have included a direct neural-behavioural investigation).

Behavioural data on motor difficulties in infants and children with WS, whilst also limited, provides further insight into the motor competencies of individuals with WS. Martin et al. (1984) reported findings from parent surveys investigating physical and cognitive development in Williams syndrome. They investigated two motor milestones in 41 children with WS and report that this group reached the motor milestone of sitting unsupported at 10.5 months and waking unsupported at 23.4 months. This contrasts with data from the World Health Organization (WHO Multicentre Growth Reference Study Group and de Onis 2006) which suggest that typically developing infants reach the sitting unsupported milestone at around 3.8-9.2 months and typically developing children will walk unsupported at around 8.317.6 months. Carrasco et al. (2005), also using parent report, state that children with WS often reach motor milestones much later than chronological age-matched typically developing children. However, the data on motor milestones remains limited in WS, as these authors only examined three motor milestones (head support, sitting without support, and walking without support). They found that while some children with WS were reaching these motor milestones at the same rate as their typically developing peers of the same age, many took much longer to achieve the milestone.

It is possible that variance in motor impairment in WS is related to variance in cognitive ability, with those who show poorer cognitive skills also showing worse motor abilities. 
There is research suggesting that individuals with intellectual disabilities are more likely to have motor impairments. That is, Smits-Engelsman and Hill (2012) observed that, in their sample, $19 \%$ of the variance in motor ability was explained by IQ scores, and for each SD lower IQ, there was an average loss of 10 percentile motor points on the Movement Assessment Battery for Children (M-ABC; Henderson et al. 2007). Therefore, motor milestone achievement might, to some extent, depend on the cognitive ability of the individuals in each sample.

There have been two studies that have investigated the wider motor profile of children with WS (Tsai et al. 2008; Wuang and Tsai 2017). Tsai et al. (2008) investigated the motor ability of eleven individuals with WS, using the Bayley Scales of Infant Development (Bayley 1993) for those who were under 42 months $(N=7)$ and the BruininksOseretsky Test (BOT) short form (Bruininks 1978) for those over 42 months $(N=4)$. Tsai et al. (2008) report that all the children with WS showed a significant deficit in both their fine and gross motor abilities (although note the small sample size). However, they did not report any information regarding the motor profile (i.e. the specific relative strengths and weaknesses) of their WS group. Wuang and Tsai (2017) investigated motor ability in thirty-eight children with WS aged 6 to 12 years using the Bruininks-Oseretsky Test of motor proficiency, second edition (BOT-2; Bruininks and Bruininks 2005). Consistent with Tsai et al. (2008), all the children presented with impairment on fine motor measures. However, only $60 \%$ of the children scored in the impaired range on the gross motor measures, with running speed and agility (hopping in place on one leg and walking on a straight line) being, on average, the most impaired. One study by Braddick and Atkinson (2013) also suggests that children with WS show a delay of up to 2 years on manual ball skills on the M-ABC (Henderson et al. 2007). Atkinson (2017) expanded on this study more and presented further findings for 24 children and adolescence with WS (aged 6.5 to 15 years), reporting that these individuals show a delay on manual dexterity, manual ball skills and static and dynamic balance on the M-ABC (Henderson et al. 2007), and many do not reach the level of a typically developing 4-year old on these tasks. Taken together, these studies suggest that individuals with WS show fine motor impairment in early childhood, and into later childhood, and there is some evidence to suggest that gross motor ability may also show impairment in early childhood and on certain gross motor tasks later in childhood (e.g. running speed and agility). Investigating the motor profile in more detail and gaining more information about where the strengths and weaknesses in the motor profile are for people with WS could help to inform intervention to improve motor abilities in this population.

There is also a significant association between level of physical activity and motor ability in typically developing children and adolescents, with those who are more involved in sports and exercise receiving higher scores on motor tasks (Barnett et al. 2008; Stodden et al. 2008; Ulrich 1987). Research suggests that, at least in younger children, the amount of physical activity in which a child engages influences motor skill development by promoting the development of motor brain areas (Becker et al. 2004; Stodden et al. 2008). The only investigation into physical activity in WS to our knowledge is the study by Nordstrøm et al. (2013), who used accelerometers to measure physical activity levels in adults with WS $(N=28)$, Down syndrome (DS) $(N=40)$, and Prader-Willi syndrome (PWS) $(N=28)$. They report that across all three groups the majority of the day was spent in sedentary activities and that only $12 \%$ of the whole sample met the Nordic recommendation for the amount of daily physical activity ( $3.5 \mathrm{~h}$ of moderate-intensity physical activity per week: Rasmussen et al. 2012). However, the authors did not measure motor ability in their sample, so we do not know whether there is an association between this low level of physical activity and potential motor deficits in WS. If there is a relationship between motor ability and involvement in sports and exercise, it may be that increasing involvement in physical activity could have positive effects on motor ability in those with WS. Alternatively, it might be the case that interventions to improve motor skills will increase confidence, accessibility, and enjoyment of taking part in physical activity, leading to better health outcomes. This potential association between motor skills and physical activity will be investigated in the following study.

The aim of the current study is to investigate the level of motor achievement that is reached by adulthood in WS, focusing particularly on the motor profile of individuals with WS. This study will examine whether the strengths and weaknesses in specific areas of fine and gross motor ability observed in childhood in WS persist into older childhood and adulthood. This study focuses on investigating not only motor behaviour per se, but also the association of physical activity with motor ability. Further to this, we employ a retrospective parent report measure regarding the achievement of a broad range of motor milestones, which adds to the limited data in the literature with respect to motor milestone achievement in WS.

\section{Method}

\section{Participants}

Power analysis using $\mathrm{G}^{*}$ Power was conducted for each planned analysis with power $=0.80$ and alpha $=0.05$ to determine sample size. The largest sample size suggested was $N=84$ participants for each group. However, given the nature of working with rare groups, we were not able to reach this 
sample size. The sample included 36 participants with a positive clinical diagnosis of WS; this is a large sample relative to other studies that have worked with this population. WS participants were aged 12-50 years and recruited via the Williams Syndrome Foundation, UK. A control sample of 40 typically developing (TD) children aged 4 to 7 years was also tested. They were divided into two groups, a TD 4- to 5year-old group and a TD 6- to 7-year-old group (henceforth referred to as TD4-5 and TD6-7 respectively), recruited from two primary schools in the London area. All participants had normal or corrected to normal vision. The age range of the typically developing children was chosen to span the hypothesised range of motor abilities of the WS group.

Participants were assessed on their verbal and non-verbal IQ using the British Picture Vocabulary Scale III (BPVS III) (Dunn et al. 2009) and the matrices subtest of the British Abilities Scales II (BAS III) (Elliott et al. 1996). Sixteen participants with WS completed the Ravens Coloured Progressive Matrices (RCPM) (Raven et al. 2003) instead of the BAS III Matrices subtest. This is due to data being collected at two different time points and we found that, when participants were tested using the BAS III Matrices, many participants were getting a raw score of 2 or 3 , indicative of reduced sensitivity of this measure for individuals with low non-verbal ability. The RCPM is a more sensitive measure and shows more variability in performance at this level of ability. Participant details are shown in Table 1. Raw scores are reported for the BPVS III and RCPM. Ability scores were derived from the raw scores of the BAS III Matrices, which gave a score based on what item in the assessment each participant began (equivalent to raw scores).

Analysis of variance (ANOVA) was conducted separately for BPVS III and BAS III matrices scores with group (3 levels; WS, TD4-5, and TD6-7) as the between-participant factor. This demonstrated the characteristic of the WS uneven cognitive profile. That is, the WS group performed significantly better on the BPVS III than both typically developing groups $\left(F_{(2,75)}=18.98, p<.001, \eta_{\mathrm{p}}^{2}=.342\right)$, and below both typically developing groups on the BAS II matrices subtest $\left(F_{(2,57)}=\right.$
$16.03, p<.001, \eta_{\mathrm{p}}^{2}=.421$ ) (see Table 1 ). For the 16 participants with WS who completed the RCPM, TD comparison data was not available. Age equivalent data, based on the standardised sample from the manual, demonstrated a mean age equivalent of 5 years (range, $<4-8$ years) for this group. This, compared with their BPVS III scores, also reflects the WS cognitive profile (Table 1).

\section{Procedures}

Ethical approval was obtained from the UCL, Institute of Education ethics committee before testing began. Typically developing participants were tested in a quiet room at their school during the day or, for a small sample of the 4-year olds, in their own home. Participants with WS were tested either in a quiet room at the University or in their own home. For the individuals with WS, the entire testing session lasted between $1 \mathrm{~h} 30 \mathrm{~min}$ and $2 \mathrm{~h}$ with breaks. For the TD children, testing was completed over four 30-min sessions for the 4-, 5-, and 6year olds, and two 1-h sessions for the 7-year olds. All participants were given breaks when needed, and for some of the 4and 5-year olds, sessions were split into 15 -min sections to reduce fatigue and maximise motivation.

\section{Measures}

Motor Milestones In order to investigate the age at which individuals with WS reached their motor milestones, parents were given a questionnaire (developed and used by Sumner et al. 2016, based on Brouwer et al. 2006). This comprised a list of 12 motor milestones (e.g. sitting without support, walking with assistance) and parents were asked what age in months their child was when these milestones were reached. This questionnaire was not given to the parents of the TD children, so the TD data reported in Sumner et al. (2016) and Farran et al. (2020) is used as a TD comparison (Table 2).

Motor Ability Motor ability was assessed using the BruininksOseretsky Test of Motor Proficiency, second edition short

\begin{tabular}{llll}
\hline Group & & & \\
\hline Mean age (years;months) (range) & WS $(N=36)$ & TD4-5 $(N=20)$ & TD6-7 $(N=20)$ \\
& $23 ; 9(8 ; 08-50 ; 07)$ & $4 ; 6(4 ; 0-5 ; 9)$ & $6 ; 6(6 ; 10-7 ; 7)$ \\
Gender F:M & $13: 7$ & $11: 9$ & $9: 11$ \\
BPVS-III $^{1}$ raw score & $120.31(62-160)$ & $83.30(57-124)$ & $105.10(72-129)$ \\
BAS-III $^{2}$ ability score & $N=20,47.75(23-103)$ & $66.55(23-107)$ & $92.20(37-131)$ \\
RCPM $^{3}$ raw score & $N=16,17.75(5-30)$ & $\mathrm{X}$ & $\mathrm{X}$ \\
\hline${ }^{1}$ British Picture Vocabulary Scale, Third Edition $^{2}$ & & \\
${ }^{2}$ British Abilities Scale, Third Edition (matrices subtest only) & & \\
${ }^{3}$ Ravens Coloured Progressive Matrices & &
\end{tabular}


form (BOT2-SF; Bruininks and Bruininks 2005). This measure was designed for typically developing people aged 3 to 21 years and was selected because the BOT-2 has high reliability (inter-rater reliability, > .90; test-retest reliability, > .80) and validity (ability to distinguish between clinical and nonclinical groups: clinical groups scored lower than non-clinical groups on three studies reported in the BOT-2 Manual, $p<.001$ ) (Deitz et al. 2007). It is also a comprehensive approach to testing specific motor skills, and it is used clinically by occupational therapists (Deitz et al. 2007).

The fine motor subtests of the BOT2-SF are as follows: fine motor precision comprised the crooked line path task, where participants were asked to draw a line through a path from a picture of a car to a picture of a house, and the folding task, where participants were asked to fold a piece of paper on the lines. Fine motor integration comprised the square task, where participants were asked to copy a picture of a square by drawing the square on paper with a red pencil, and the star task, where participants were asked to copy a picture of a star by drawing it on paper using a red pencil. Finally, manual dexterity was measured with the transferring pennies task, where participants had to pick up plastic pennies, move them from one hand to another, and drop them into a pot as quickly as possible.

The gross motor subtests of the BOT2-SF are as follows: bilateral co-ordination which comprised the tapping task, where participants were asked to alternatively tap their fingers and feet on the same side of the body at the same time to a rhythm, and the jumping in place task, where participants were asked to put the same arm and leg in front/behind them and then jump to switch the arms and legs around so the other arm and leg were in front. Running speed and agility comprised the hopping in place task, where participants were asked to hop in place on one foot for $15 \mathrm{~s}$, and the walking on a line task, where they had to walk forward six steps on a line. The balancing task required them to balance on a balance beam on one leg for $10 \mathrm{~s}$ while looking at a red target, placed at eye level, $10 \mathrm{ft}$ in front of them. Upper limb control involved the dropping and catching task, where they had to drop and catch a tennis ball 5 times, and the dribbling task, where they had to drop the ball with one hand, and then dribble it with alternate hands 10 times. Finally, Strength comprised the sit-ups task, where they were asked to do sit-ups for $30 \mathrm{~s}$, and the push-up task, where they were asked to do knee push-ups (i.e. where they were asked to adopt a 'hands and knees' position, with the legs bent at the knee, feet crossed) for $30 \mathrm{~s}$.

Physical Activity All participants were given a questionnaire regarding their level of physical activity. The experimenter read the questions to the participants, participants responded verbally, and the experimenter noted their response. Participants were asked, in a typical week, how many times during weekdays and during the weekends they participated in sports (excluding compulsory P.E. as the majority of the WS group were adults and therefore did not take part in any compulsory exercise). The experimenter gave prompts where

Table 2 Mean (range) of motor milestone achievement (in months) for TD and WS, with the World Health Organization (WHO Multicentre Growth Reference Study Group and de Onis 2006) percentiles where applicable

\begin{tabular}{|c|c|c|c|c|c|c|c|c|c|c|}
\hline & \multicolumn{2}{|c|}{$\begin{array}{l}\text { WHO } \\
\text { Age in months at which } \\
\text { milestone achieved }\end{array}$} & \multirow{2}{*}{\multicolumn{4}{|c|}{$\begin{array}{l}\text { TD group (from Sumner et al. } 2016 \text { and } \\
\text { Farran et al. 2020) } \\
\text { Mean (range) age years:months } \\
9.11 \text { years }(7.5-10.74)\end{array}$}} & \multicolumn{4}{|c|}{ WS group } \\
\hline & & & & & & & \multicolumn{4}{|c|}{$\begin{array}{l}\text { Mean (range) age years:months } \\
25.96(9.3-50.67)\end{array}$} \\
\hline & $\mathrm{M}(\mathrm{SD})$ & Range & $N$ & $\mathrm{M}(\mathrm{SD})$ & Percentile & Range & $N$ & $\mathrm{M}(\mathrm{SD})$ & Percentile & Range \\
\hline Lift head & & & 27 & $2.06(1.30)$ & & $0.2-5$ & 6 & $5.83(4.67)$ & & $2-12$ \\
\hline Turn back to belly & & & 27 & $4.29(1.74)$ & & $0.8-9$ & 6 & $7.17(4.26)$ & & $3-14$ \\
\hline Sit without support & $6(1.1)$ & $3.8-9.2$ & 30 & $5.65(1.58)$ & 50 th & $2-9.5$ & 12 & $10.67(5.77)$ & $>99$ th & $3-24$ \\
\hline Crawl hands and knee & $8.5(1.7)$ & $5.2-11.4$ & 30 & $7.85(1.98)$ & 25 th & $5-12$ & 9 & $13.33(5.52)$ & $>99$ th & $5-21$ \\
\hline Stand with assistance & $7.6(1.4)$ & $4.8-11.4$ & 29 & $8.60(1.75)$ & 75 th & $4-11$ & 10 & $14.90(5.53)$ & $>99$ th & $10-24$ \\
\hline Stand without support & $11.0(1.9)$ & $6.9-16.9$ & 32 & $10.73(1.28)$ & 50 th & $7-14$ & 8 & $20.88(8.77)$ & $>99$ th & $12-36$ \\
\hline Walk with assistance & $9.2(1.5)$ & $6-13.7$ & 32 & $11.17(2.06)$ & 90th & $6-16$ & 9 & $19.00(8.02)$ & $>99$ th & $12-36$ \\
\hline Walk without support & $12.1(1.8)$ & $8.2-17.6$ & 33 & $13.03(1.91)$ & 75 th & $9.5-18$ & 16 & $23.31(8.50)$ & $>99$ th & $15-42$ \\
\hline Walk up/down stairs with support & & & 31 & $12.76(4.22)$ & & $6-24$ & 8 & $30.63(12.68)$ & & $12-48$ \\
\hline Walk up/down stairs without support & & & 30 & $20.77(9.55)$ & & $10-60$ & 10 & $49.90(27.74)$ & & $18-108$ \\
\hline Ride a bike with stabilisers & & & 32 & $33.56(10.74)$ & & $18-60$ & 14 & $84.43(59.56)$ & & $24-216$ \\
\hline Ride a bike without & & & 30 & $57.23(14.49)$ & & $30-96$ & 7 & $120.86(103.95)$ & & $54-348$ \\
\hline
\end{tabular}


needed, such as 'did you do any sports or exercises yesterday?' and 'are you going to be doing any sports or exercise after school today/over the weekend?'. Participants were also given some examples of types of physical activity to aid recollection, such as 'dancing, playing football, going to the gym, etc.' The maximum score for this questionnaire was eight, and this was computed by adding up the number of activities taken part in on weekdays and weekends (max score of 4 for each), with each activity being a separate score, regardless of the length of activity. The maximum score was given if participants reported that they took part in 5 or more physical activities on weekdays and 5 or more physical activities weekends.

\section{Data Analyses}

Motor milestone data was collected retrospectively, and as such, there was missing data where parents left cells blank when unsure. Due to the low numbers of responses, only descriptive statistics of this data are presented as there was not enough power to perform meaningful statistical analyses. Assumptions of normality were investigated using Kolmogorov-Smirnov tests. It was found that the data met assumptions of normality for the majority of variables on both the BOT2-SF and the physical activity questionnaire (Kolomorov-Smirnov, $p \geq .05$ ), and that outliers were not significantly affecting the means of the data when the 5\% trimmed mean was looked at. Therefore, parametric tests were conducted. To investigate the association between motor ability and age, correlations were conducted between BOT2-SF score and age. Correlations were also conducted between BPVS III score and BOT2-SF score to check for any associations between IQ and motor ability. To consider the general level of motor achievement in WS, a one-way ANOVA was conducted on the total motor raw scores for the BOT2-SF, with group (WS, TD4-5, TD6-7) as a between-participant factor, followed up using Tukey's pairwise comparison tests. Next, the mean and SD of the TD4-5 group were used to calculate $z$-scores of performance from the raw scores of performance on each subdomain of the BOT2-SF for the WS group. Therefore, to determine the motor profile of the WS group, a one-factor ANOVA of the z-scores was carried out on the WS data only with subtest (8 levels: fine motor precision, fine motor integration, manual dexterity, bilateral coordination, balance, running speed and agility, upper limb control, and strength) as the within-participant factor. To examine the amount of physical activity that the individuals with WS took part in, a one-way ANOVA was conducted to compare performance across the groups on the level of physical activity involvement (Max. score, 8). Finally, Pearson's bivariate correlations and partial correlational analyses (controlling for age) between motor ability (BOT2-SF) and physical activity were conducted.

\section{Results}

\section{Motor Milestones}

Due to the low numbers of responses on account of missing data, only descriptive statistics of this data are presented. The mean age of milestone achievement in months is presented in Table 2, along with the number of participant responses for each milestone and the range of ages for each milestone. Six of the 12 milestones can be compared with the World Health Organization (WHO) guidelines for when children typically reach a range of motor milestones (WHO Multicentre Growth Reference Study Group and de Onis 2006). The remaining six can be compared with the data from the TD sample reported in Sumner et al. (2016) and Farran et al. (2020), with higher percentiles indicating later achievement of the milestone. The WS group is performing higher than the 99th percentile for all WHO motor milestones, indicating a significant delay in reaching these milestones, and later than the TD sample for the remaining motor milestones. It should be noted that in each case one or more participants with WS were reported to have achieved that motor milestone within the range reported in Sumner et al.'s (2016) typical group, indicative of a broad range of motor milestone achievement in WS. Despite this, overall, the findings suggest that individuals with WS are showing delays in reaching all motor milestones investigated, although the small sample size should be noted.

\section{Motor Ability}

\section{The Effect of Age on Motor Performance}

Research consistently demonstrates that chronological age is rarely related to cognitive impairment in WS (KamirloffSmith 1998). Equally, the age range of our WS group was likely past the age where age-related motor development might be expected. Correlations were conducted to determine the relationship between age and motor performance in this group, but as expected, chronological age was not related to motor ability in the WS group. There were, however, correlations between chronological age and motor ability in the TD group, which would be expected as the motor ability is expected to improve with age (Table 3). As such, all further correlations were carried out with chronological age partialled out (Table 4). Whilst this was not strictly necessary for the WS group, given the medium effect size for the motor-age association in this group, we considered the most cautious approach was to partial out chronological age for both groups.

\section{The Effect of IQ on Motor Ability}

Research has demonstrated a relationship between cognitive ability and motor ability in populations with intellectual 
disability (Smits-Engelsman and Hill 2012). To investigate whether this was also the case in our group of individuals with WS, partial correlations (controlling for age) were performed between raw BPVS III score and total motor ability. A relationship was found between motor ability and BPVS III score in the WS group. However, this was not the case in the TD group.

\section{BOT2-SF Motor Performance Zones}

To further investigate the motor percentiles that individuals with WS fell into in relation to the general population, standard scores were derived from the BOT-2 manual for the TD and WS groups. For participants in the WS group who were over 21 years $(N=25)$, the maximum adult age of 21 was used to calculate the standard score. The percentiles here work in the opposite way to the WHO motor milestone percentiles, i.e. the lower the percentile, the worse the performance is on motor tasks. Results indicate that most of the WS group were performing in the 'below average' (3rd-16th percentile) or 'well below average' $(<2$ nd percentile) zone of the BOT2SF. However, two participants were scoring in the 'average' zone (17th-83rd percentile), but no WS participants scored above the 46th percentile. The TD children were all performing in the 'average', 'above average' (83rd-97th percentile), and 'well above average' ( $>98$ th percentile) zones (Fig. 1).

\section{BOT2-SF Total Motor Abilities}

To consider the general level of motor achievement in WS, a one-way ANOVA was conducted on the total motor raw scores for the BOT2-SF, with group (WS, TD4-5, TD6-7) as a between-participant factor (Table 5). This demonstrated an effect of group $\left(F_{(2,75)}=14.34, p<.001, \eta^{2}=.282\right)$. Post-hoc Tukey's tests indicated that the WS group was performing at the TD4-5 level $(p>.05)$, and both the WS and TD4-5 groups were performing below the TD6-7 level ( $p<.05$ for both). Therefore, the WS group is matched as a group to the TD45 group in terms of overall motor abilities.

As shown above, the TD 4-5-year-old group was not significantly different from the WS group on overall motor ability, and so the mean and SD of the TD4-5 group were used to calculate $z$-scores of performance from the raw scores of performance on each subdomain of the BOT2-SF for the WS

Table 3 Correlations between chronological age and raw total motor scores for the WS and TD groups. Italics indicate significant correlations

\begin{tabular}{ll}
\hline Group & Age $\times$ total motor score \\
\hline WS $(n=36)$ & $r=.323, p=.054$ \\
$\mathrm{TD}(n=40)$ & $r=.806, p<.001$
\end{tabular}

group. This enables us to determine the profile of scores of the WS group relative to the profile that would be expected for their overall level of motor ability. A one-factor ANOVA of the $z$-scores was carried out on the WS data only (note that the 4-5-year-old data by design was a flat line) with subtest ( 8 levels: fine motor precision, fine motor integration, manual dexterity, bilateral coordination, balance, running speed and agility, upper limb control, and strength) as the withinparticipant factor (Table 6; Fig. 2).

Results demonstrated an atypical profile of motor abilities, shown by a main effect of subtest $\left(F_{(1,35)}=55.58, p<.001\right.$, $\eta^{2}=.612$ ). Post-hoc Sidak corrected $t$ tests demonstrated that this was due to a relative strength in the subdomain of 'upper limb control', on which participants with WS scored significantly higher than all other subtests $(p<.05$ for all), and a relative weakness in 'balance', on which participants with WS scored significantly lower on than all subtests, except fine motor integration $(p=.184)$ (all other comparisons, $p<.05$ ). We also wanted to see whether the WS group showed a strength in upper limb control in comparison with older TD children, so an independent samples $t$ test was performed to look for differences between the WS and TD6-7 group on upper limb control. The mean raw score on upper limb control for the WS group was 7.47 (SD, 2.63; range, 12.00); for the TD4-5 group, it was 2.50 (SD, 2.01; range, 6.00); and for the TD6-7 group, it was 6.75 (SD, 2.63; range, 11.00). While the WS group showed a relative strength in upper limb control compared with the TD 4-5-year olds (Cohen's $d=2.32$ ) and within their motor profile, they did not perform significantly better than the TD 6-7-year olds ( $p=.895$; Cohen's $d=$ 0.293 ), suggesting that their upper limb control ability is still significantly delayed for their chronological age.

\section{Physical Activity}

To examine the amount of physical activity that the individuals with WS took part in, a one-way ANOVA was conducted to compare performance between the groups on the level of sports and physical activity involvement (Max. score, 8) (Table 7). This highlighted significant differences across the groups $\left(F_{(2,75)}=6.02, p=.004, \eta^{2}=.142\right)$. Post-hoc Tukey's comparisons indicated that the WS group was taking part in the same amount of physical activity as the TD 4-5-year olds $(p=.609)$, but less than the $6-7$-year olds $(p=.003)$. There

Table 4 Partial correlations (chronological age partialled out) between raw BPVS score and raw total motor scores for the WS and TD groups

\begin{tabular}{ll}
\hline Group & BPVS III $\times$ total motor score \\
\hline WS $(n=36)$ & $r=.668, p=<.001$ \\
$\mathrm{TD}(n=40)$ & $r=.251, p=.124$ \\
\hline
\end{tabular}


Fig. 1 Distribution of BOT2-SF 'zones' in the WS, TD4-5, and TD6-7 groups

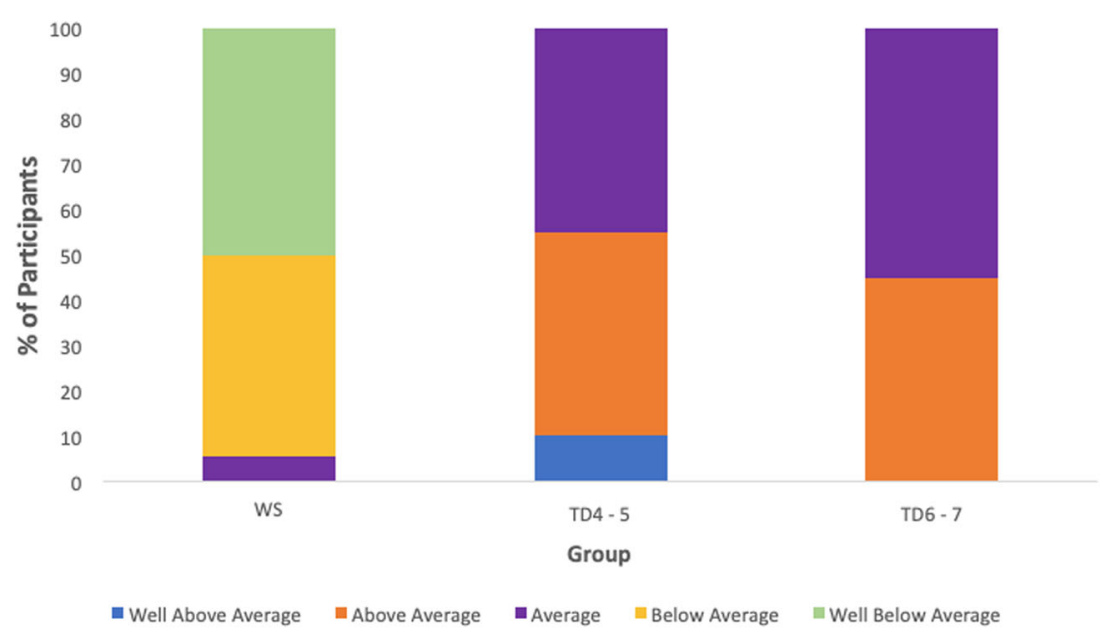

was no significant difference between the amount of physical activity that the TD4-5 and TD6-7 groups took part in $(p=.119)$.

\section{Correlations Between Motor Ability and Physical Activity}

Partial correlational analyses, controlling for age, were performed to investigate the potential relationship between motor ability and participation in physical activity in typical development and in WS (Table 8). To do this, the two TD groups were combined. As there were two correlations per group, a critical alpha of $p \leq .025$ was used. A significant partial correlation was observed between fine motor ability and physical activity for the TD group, but not the WS group. There were no other significant correlations between motor ability and physical activity in either group.

\section{Discussion}

We examined motor abilities in individuals with WS and typically developing children and how these motor abilities related to participation in physical activity. We report a substantial delay in achieving motor milestones in WS. Furthermore, the hypothesised motor deficits were observed in the WS group, with this group scoring at the level of a 4- to 5-year-old typical

Table 5 Mean (range) participant raw total motor score on BOT2-SF

\begin{tabular}{ll}
\hline Group & BOT2-SF ${ }^{1}$ mean raw score (range) \\
\hline WS $(N=36)$ & $44.86(12-76)$ \\
TD4-5 $(N=20)$ & $47.05(31-63)$ \\
TD6-7 $(N=20)$ & $63.45(50-74)$
\end{tabular}

\footnotetext{
${ }^{1}$ Bruininks-Oseretsky Test of Motor Proficiency short form, second edition
}

child. Interestingly, individuals with WS showed a relative strength in upper limb control, a subtest that required the participant to drop and catch and also to dribble a tennis ball. Note, however, that the performance of the WS group in this subdomain was still only at the level of a typically developing 6- to 7-year old. The WS group showed a particular weakness in balance (i.e. they performed below the level of a typically developing 4- to 5-year old), which is likely due to cerebellar abnormalities (Jernigan and Bellugi 1990; Jernigan et al. 1993; Jones et al. 2002; Reiss et al. 2000), alongside other factors which are discussed further below. The hypothesis that individuals with better motor abilities would also be involved in more physical activity (or vice versa) was partially supported in the TD group, but not in the WS group, as neither fine nor gross motor ability correlated with participation in physical activity in this group. This will be discussed in detail below.

\section{Motor Milestones in WS}

Previous findings investigated two or three motor milestones in WS (Martin et al. 1984; Carrasco et al. 2005), reporting substantial delay. In line with these studies, our extensive set of twelve motor milestones support this finding. Individuals with WS in our sample were reported to have substantial delays in reaching all motor milestones, scoring above the 99th percentile for the six motor milestones that could be compared with the WHO standards (WHO Multicentre Growth Reference Study Group and de Onis 2006) and achieved milestones later than a TD sample for the remaining six milestones. However, it is important to note that the range of milestone achievement of the WS group often overlapped with that expected in typical development, and that a small number of respondents contributed to this dataset. Parents were asked to fill in the motor milestones questionnaire retrospectively, and given the age range of the participants, many parents noted that they could not remember when their son or daughter was 
Table 6 Mean (range) raw score for each of the 8 BOT2-SF subdomains for each group

\begin{tabular}{|c|c|c|c|c|c|c|c|c|}
\hline & \multicolumn{3}{|c|}{ BOT2-SF fine motor subdomain } & \multicolumn{5}{|c|}{ BOT2-SF gross motor subdomain } \\
\hline & FMP & FMI & MD & BLC & Balance & RSA & ULC & Strength \\
\hline WS & $7.19(0-14)$ & $5.64(0-10)$ & $2.67(1-5)$ & $4.64(0-9)$ & $4.14(0-10)$ & $5.47(0-9)$ & $7.47(0-12)$ & $7.69(1-15)$ \\
\hline TD4-5 & $7.40(4-10)$ & $8.00(4-10)$ & $2.80(1-4)$ & $5.60(0-7)$ & $6.70(3-8)$ & $6.55(2-9)$ & $2.50(0-6)$ & $7.55(3-14)$ \\
\hline TD6-7 & $11.30(8-14)$ & $9.55(8-10)$ & $4.00(2-5)$ & $6.90(5-9)$ & $7.65(6-8)$ & $8.15(5-9)$ & $6.75(1-12)$ & $9.75(7-15)$ \\
\hline
\end{tabular}

$F M P$, fine motor precision; $F M I$, fine motor integration; $M D$, manual dexterity

able to reach certain milestones. Further research is required either by collecting concurrent motor milestone data as milestones emerge (although this is difficult due to often late diagnosis of WS), or by asking parents of younger participants with WS for retrospective milestone information. Nonetheless, our data indicate that motor difficulties are apparent in WS from the outset.

\section{Motor Skills in WS}

This study examined the profile of motor deficits beyond childhood in the WS population. We demonstrated that the fine and gross motor difficulties observed in children with WS by Atkinson (2017), Tsai et al. (2008), and Wuang and Tsai (2017) persist into older childhood and adulthood. Overall, our WS sample (aged 12 to 50 years) demonstrated motor performance at the level of a TD 4 to 5 years old. This severity of motor problems suggests that motor ability in WS could be affecting other aspects of the individuals' lives. For example, although not assessed in this study, this raises the possibility that the motor deficit in WS might have cascading impacts on other domains such as language, spatial ability, and social cognition. At a practical level, if an individual is less confident with their movement, then they might also be less able and less likely to want to independently perform tasks of daily living, such as getting dressed independently, or cooking a meal, an area of functioning that individuals with WS are known to struggle with (Dilts et al. 1990; Greer et al. 1997; Gosch and Pankau 1994; Mervis et al. 2001; Udwin 1990).

Interestingly, like the WS cognitive profile (Morris and Mervis 1999), the motor profile of individuals with WS was highly varied across tasks. That is, individuals performed relatively well in the subdomain of upper limb control (at the level of 6 to 7 year olds) but performed relatively poorly on the balance subdomain (below the level of 4 to 5 year olds) within their own motor profile (using the motor profile of 4- to 5-year olds as a benchmark).

Anecdotally, many participants reported enjoying the upper limb control task. Taking a dynamical systems theory (DST; Thelen and Smith 1998) approach, this might be due to familiarity of the task, i.e. many of the participants were likely to have played with a ball before at some point in their life and so it may have appeared to be a less threatening task. Of course, this would suggest that this relative strength is not necessarily specific to WS, and thus, it is likely that other syndrome-specific processes also contributed to their performance on this task. The kinds of ball skills measured in the upper limb control tasks are akin to those measured in the manual ball skills subdomain of the M-ABC (Henderson et al. 2007), skills that have not been reported as a particular strength in children with WS (Atkinson 2017; Braddick and Atkinson 2013). It might be that this is a skill that improves over time (our sample was older than the sample reported by Atkinson 2017 and Braddick and Atkinson 2013) or this might just reflect the relative sensitivity of the subdomains of the M-ABC vs. the BOT2-SF. Indeed, many children in Atkinson's (2017) study fell within the 6- to 7-year-old range of performance for this task, which is akin to the level of ability of the current sample.

The task within the balance subdomain was to balance on a thin beam on one leg for $10 \mathrm{~s}$. However, the majority of individuals were unable to balance on one leg for more than a second. These difficulties in balance are consistent with findings from Atkinson (2017), who found that children and adolescents with WS showed deficits on both static and dynamic balance tasks from the M-ABC (Henderson et al. 2007) and, in most cases, did not attain a 4-year-old age equivalent (the minimum score on the M-ABC). However, Tsai et al. (2008)
Fig. 2 Profile of motor abilities in WS (purple line): $z$-scores based on the mean and standard deviation of TD 4-5-year olds (matched for overall level of motor ability)

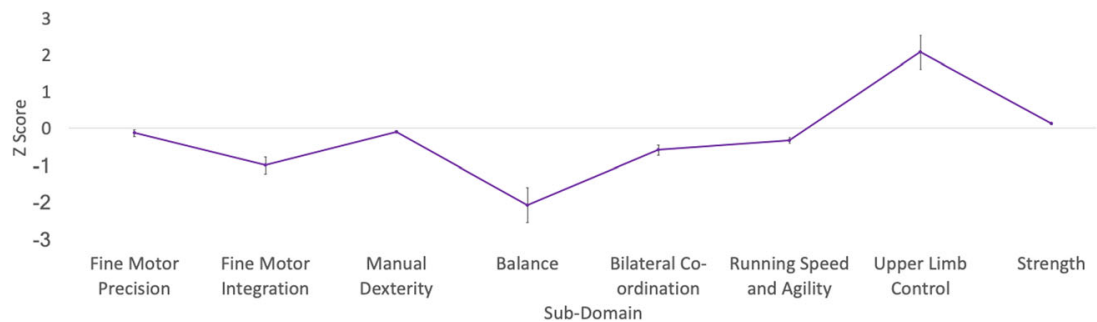


report half of their sample of children with WS could achieve the full score (6) on the BOT2-SF (Bruininks and Bruininks 2005) Balance subdomain. Note that there were only 4 children who performed this task, and so while half the sample (2 participants) scored full marks, the other half of the children (2 participants) scored only a 1 and 2 out of 6 . Furthermore, Wuang and Tsai (2017) report that $57.9 \%$ of their sample showed an impairment (defined as more than 1.5 SD below the normative mean) on the balance task. Deficits in balance in WS may be partially due to atypicalities of the cerebellum, which are often reported in this population (Jernigan et al. 1993; Osório et al. 2014; Reiss et al. 2000). The cerebellum is arguably the main structure in the brain responsible for balance. However, taking into account the DST (Thelen and Smith 1998), other factors, such as fear and low motivation, may have played a role in the difficulties that these individuals are showing on this balance task.

Other motor brain areas that have been found to show structural differences in WS could also be contributing to the low motor scores. For example, the basal ganglia are involved with the planning and control of voluntary motor acts (Monchi et al. 2006), and these structures have often been shown to be structurally atypical in WS (Jernigan et al. 1993; Reiss et al. 2000). Indeed, Cowie et al. (2012) and Hocking et al. (2011) report that motor planning is impaired in individuals with WS, with this population showing difficulty scaling movement to step height. In several of the BOT2-SF tasks, the instructions that are given to participants involve several steps that must be followed to receive the points available. For example, on the 'pennies' task, participants must only move one penny at a time with one hand, transfer it to the other hand, and then place it in a pot. It is possible, therefore, that a motor planning deficit is contributing to their poor performance on such tasks.

A general contributing factor to poor motor ability in WS is IQ. A relationship is reported between cognitive ability and motor ability in other populations with intellectual disability. This relationship was broadly replicated in our WS sample, as demonstrated by the relationship between BPVS III raw score (a proxy for IQ) and motor ability in this group. SmitsEngelsman and Hill (2012) found that variance in motor ability in groups with and without motor difficulties could be explained by IQ in 19\% of cases. Furthermore, for each standard deviation lower in IQ, a mean loss of 10 percentile points was observed. It has been found that IQ for individuals with

Table 7 Mean (SD) participant score on the Physical Activity Questionnaire (Max. score, 8)

\begin{tabular}{llll}
\hline & \multicolumn{2}{l}{ Group } & \\
\cline { 2 - 4 } & WS $(N=36)$ & TD4-5 $(N=20)$ & TD6-7 $(N=20)$ \\
\hline Physical activity score & $2.31(1.62)$ & $2.85(1.39)$ & $4.0(2.25)$ \\
\hline
\end{tabular}

WS typically ranges from 55 to 62 (Greer et al. 1997; Morris and Mervis 1999), and using this information, we can predict from Smits-Engelsman and Hill (2012) that the motor percentile for individuals with WS should be around the 10th percentile, which on the BOT2-SF motor zones would put them in the 'below average' zone. Exploring this categorically, 16/ 36 of the participants fell into this zone. The remaining participants with WS either fell in the average zone $(2 / 36 ; 17$ th83 rd percentile) or the 'well below average' zone (18/36), which is below the 2 nd percentile. This indicates that many individuals with WS are performing lower than would be predicted for their cognitive abilities. Thus, whilst our finding of a positive relationship between total motor ability and raw score on the BPVS III suggests that the motor difficulties in WS are at least in part an artefact of having learning difficulties, it appears that there are syndrome-specific factors over and above their low IQ which further negatively impact motor ability in this group.

Related to the general relationship between motor skills and cognitive ability, as discussed in the introduction, poor motor skills may also have a downstream impact on the development of specific cognitive skills, such as spatial abilities. For individuals with WS, this particular relationship deserves some discussion, given that impaired spatial performance is a hallmark characteristic of the WS phenotype (e.g. Broadbent et al. 2014; Farran and Jarrold 2003; Farran et al. 2001). Several studies have demonstrated an association between motor ability and spatial skills in typically developing children (e.g. Clearfield 2004; Frick and Möhring 2013; Schwarzer et al. 2013). Overall, findings suggest a strong relationship between motor abilities and spatial skills in infancy in the typical population and perhaps suggests that one of the reasons that individuals with WS show such severe deficits in their spatial skills (e.g. Broadbent et al. 2014; Farran and Jarrold 2003; Farran et al. 2001, 2019) is the low motor ability (although see Farran et al. 2001). Clearly, further research is required to investigate this hypothesis empirically.

It was observed during testing that many of the WS group showed some discomfort or embarrassment during certain motor tasks, particularly the hopping, push-up and sit-up tasks, and in some cases declared that they were not able to complete the task for the required amount of time, despite performing the task correctly. In line with the DST (Thelen

Table 8 Two-tailed correlations (chronological age partialled out) between fine and gross motor ability and physical activity involvement in the WS and TD groups. Critical alpha, $p \leq .025$

\begin{tabular}{lll}
\hline & WS $(n=36)$ & TD $(n=40)$ \\
\hline Gross motor $\times$ physical activity & $r=.229, p=.186$ & $r=.087, p=.597$ \\
Fine motor $\times$ physical activity & $r=.165, p=.344$ & $r=.369, p=.021$ \\
\hline
\end{tabular}


and Smith 1998), this suggests that factors such as motivation and negative past experiences lead these individuals to be less likely to take part in physical activity due to fear of 'not being good enough'. Harter (1987) reports that people's actions are highly motivated by a need to avoid situations where they would have to show their low ability to other people, which may lead to a cycle where initial failure leads to withdrawal from the failed activity, which in turn leads to less opportunity to practise and master the skill (Schoemaker and Kalverboer 1994). Furthermore, Schoemaker and Kalverboer (1994) found that when children with poorer motor coordination were told that they would be taking part in physical activity, they were more anxious than their peers with better motor coordination. Skinner and Piek (2001) also found that 8- to 14-year olds with DCD showed increased levels of trait and state anxiety and lower self-esteem than their typically developing peers, and such traits have also been reported in adults with DCD (Hill and Brown 2013). Individuals with WS show high levels of trait anxiety (Papaeliou et al. 2012; Stinton et al. 2010; Woodruff-Borden et al. 2010), and so it is possible that there is an association between these high levels of anxiety and performance on motor tasks, and indeed motor learning. Taken together, the above studies suggest that motor impairment is likely to have wide-reaching consequences for a number of other aspects of development.

\section{Motor Ability and Physical Activity}

As found in previous studies (e.g. Barnett et al. 2008; Wrotniak et al. 2006), motor ability was associated with participation in physical activity in typically developing children. This was true for fine abilities in the TD group, but not for gross motor abilities (although note that the relationship between gross motor ability and physical activity in this group also showed a medium effect size despite being non-significant). This pattern is intriguing, as the majority of the physical activity clubs that children take part in (e.g. ballet, football) predominantly involve gross motor ability more than fine motor ability. Whilst this does question the sensitivity of the physical activity measure, it is of course possible that children with better fine motor ability are more able to perform more precise and controlled movements, making them more confident in their movements and therefore more likely to take part in physical activity. It is, however, not possible to determine the causal direction from this data. The correlation between motor ability and participation in physical activity was not significant in the WS group. This may be partly due to a lack of opportunity. Many of the TD children attended after school sports groups, whereas those in the WS group are usually not afforded the same opportunities to join sports groups, as they may not have lived in an area that provides suitable sports groups for their age and level of ability.
Importantly, the results from this study support previous genetic, neuroimaging, and behavioural findings, showing that individuals with WS show deficits in both total motor ability and in specific areas of their motor profile, particularly balance. These findings fit within the DST model (Thelen and Smith 1998), which suggests that multiple factors, both internal and external to the individual, need to be considered when performing a movement. Factors internal to the individual, such as muscle strength, flexibility, and coordination; brain structure and function, and genetics, controlling cognition, autonomic function, and motivation must be considered. External factors, such as distractions, size of objects to be manipulated, type of floor surface, and space available, also must be considered when the individual performs a movement. All these internal and external systems must work together to produce a single strategy for motor control for that particular motor task.

Taking a DST approach, we can consider motor ability with respect to the internal and external factors that influence motor ability in WS. For example, it is likely that internal factors such as differences in the structure and function of the cerebellum (Jernigan et al. 1993; Osório et al. 2014; Reiss et al. 2000, basal ganglia (Jernigan et al. 1993; Reiss et al. 2000), and ventral and dorsal stream (Chapman and Goodale 2008; Jax and Rosenbaum 2009; Schindler et al. 2004) significantly impact the motor ability of individuals with WS. Fear and motivation are other internal factors that are likely influencing success on standardised motor assessment, for example, on the balance task, due to past experiences of poor stability even on flat surfaces and less motivation to continue the task. Similarly, external factors such as space available to complete the tasks may also influence motor ability, as some individuals were tested in their own homes and some in schools or in a quiet room in the University. Whilst all spaces were suited to the space needed to complete the motor tasks, variation in the space available and the familiarity of the environment were not controlled for.

\section{Limitations and Future Research}

One limitation of this research was that the short form of the BOT-2 was used rather than the full motor assessment, meaning that we are only getting a snapshot of motor abilities. This was due to time constraints, as the short form takes only around 15-20 min to complete, whereas the full assessment takes around $1 \mathrm{~h}$ to complete. As the BOT2-SF was completed in conjunction with other assessments, and the full testing session for each study was, on average, $2 \mathrm{~h}$ in length, performing the full motor assessment would have been impractical. However, it would be interesting to get a clearer and more in-depth picture of motor abilities in WS by performing the whole motor assessment. Additionally, the full BOT-2 assessment has standardisation data for each subdomain, 
which would enable us to better examine the motor profile. Further to this, it would also be interesting to investigate the weakest area of performance, balance, in more depth. It may be that there is a difference in static vs. dynamic balance, which was not assessed during the BOT2-SF, or that there was another aspect of this balance task specifically that individuals struggled with. It would also be interesting to investigate whether differences in the cerebellum of these individuals would are associated with performance on balance tasks.

A relationship has been observed between cognitive ability and motor ability in other populations with intellectual disability (Smits-Engelsman and Hill 2012), which may be a contributing factor to explaining the similarities in the motor profile in the WS group. To test whether the presence of learning difficulties generally would lead to this motor profile, the study would need to be repeated with a population who had learning difficulties (and a similar IQ to individuals with WS: 55 to 62 (Greer et al. 1997; Morris and Mervis 1999), but no cerebellar abnormalities or hypotonia.

The physical activity questionnaire could be improved. This questionnaire asked individuals how many times a week that they engaged in physical activities. Due to the young age of the typically developing children, particularly the 4- and 5-year olds, it is possible that some of the data is not accurate, as many children did not know or could not remember. The physical activity questionnaire could also be expanded to include information on the types of activities that children were involved in, e.g. team sports (football, basketball, etc.) vs. individual activities (swimming, dancing, etc.), to see whether there would be differences in motor performance depending on the motor activity. It may also be the case that the young age of the typically developing children (4 to 7 years) may have reduced the variability in physical activity scores, as they may have not been in as many sports clubs as older children due to having less choice and independence because of their young age. This, in turn, would have weakened any correlations between motor ability and physical activity in this group.

Taken together, these findings suggest that the motor deficit previously reported in children with WS persists into adulthood. This might be affecting other aspects of the individuals' lives, such as daily living and spatial skills. Further research is needed to determine how the poor motor ability seen in this population impacts other aspects of development.

\footnotetext{
Acknowledgements A special thank you to Fatima Esfandi for her help with data collection. Thank you to Emma Sumner for sharing her questionnaire and pointing us towards her TD motor milestone descriptive statistics. Thank you to Abacus Belsize Primary School, William Patten Primary School, and the Williams Syndrome Foundation UK, for enabling this research to take place. Special thanks go to all the participants for taking part in the study. We sadly state that Annette Karmiloff-Smith passed away before this study was completed.
}

Author Contributions EF, AKS, and EH conceived the initial design of the motor aspects of the study. LAM designed and executed the study, collected the data, performed the data analyses, and wrote the paper. EKF collaborated with the design and writing of the study, as the primary supervisor of LAM. HD assisted with data collection. EKF, HD, and $\mathrm{EH}$ collaborated in the writing and editing of the final manuscript. We sadly state that AKS passed away before this study was completed. We would like to publicly acknowledge the influence of her thinking and theoretical views on writing up this study.

Funding information This research was supported by an ESRC PhD studentship and The Waterloo Foundation (Grant number: FPS 596). Hana D'Souza is a Beatrice Mary Dale Research Fellow supported by Newnham College, University of Cambridge.

\section{Compliance with Ethical Standards}

Conflict of Interest The authors declare that they have no conflict of interest.

Ethics Statement The authors obtained ethics approval from the Institutional Review Board (IRB) of the UCL Institute of Education.

Informed Consent Statement IRB-approved informed consent and assent procedures were followed for all participants in this study.

Open Access This article is licensed under a Creative Commons Attribution 4.0 International License, which permits use, sharing, adaptation, distribution and reproduction in any medium or format, as long as you give appropriate credit to the original author(s) and the source, provide a link to the Creative Commons licence, and indicate if changes were made. The images or other third party material in this article are included in the article's Creative Commons licence, unless indicated otherwise in a credit line to the material. If material is not included in the article's Creative Commons licence and your intended use is not permitted by statutory regulation or exceeds the permitted use, you will need to obtain permission directly from the copyright holder. To view a copy of this licence, visit http://creativecommons.org/licenses/by/4.0/.

\section{References}

Atkinson, J. (2017). The Davida Teller Award Lecture, 2016: visual brain development: a review of "dorsal stream vulnerability"-motion, mathematics, amblyopia, actions, and attention. Journal of Vision, 17(3), 26-26. https://doi.org/10.1167/17.3.26.

Atkinson, J., King, J., Braddick, O., Nokes, L., Anker, S., \& Braddick, F. (1997). A specific deficit of dorsal stream function in Williams' syndrome. Neuroreport, 8(8), 1919-1922. https://doi.org/10.1097/ 00001756-199705260-00025.

Barnett, L. M., Morgan, P. J., van Beurden, E., \& Beard, J. R. (2008). Perceived sports competence mediates the relationship between childhood motor skill proficiency and adolescent physical activity and fitness: a longitudinal assessment. International Journal of Behavioral Nutrition and Physical Activity, 5(1), 40. https://doi. org/10.1186/1479-5868-5-40.

Bayley, N. (1993). Bayley scales of infant development. San Antonio. TX: Psychological Corporation.

Becker, W., Lyhne, N., Pedersen, A. N., Aro, A., Fogelholm, M., Phorsdottir, I., et al. (2004). Nordic Nutrition Recommendations 2004-integrating nutrition and physical activity. Scandinavian 
Journal of Nutrition, 48(4), 178-187. https://doi.org/10.1080/ 1102680410003794.

Bellugi, U., Wang, P. P., \& Jernigan, T. L. (1994). Williams syndrome: an unusual neuropsychological profile. In S. H. Broman \& J. Grafman (Eds.), Atypical cognitive deficits in developmental disorders: implications for brain function (pp. 23-56). Hillsdale: Lawrence Erlbaum.

Bertenthal, B. I. (1996). Origins and early development of perception, action, and representation. Annual Review of Psychology, 47(1), 431-459 https://doi-org.libproxy.ucl.ac.uk/10.1146/annurev.psych. 47.1.431

Braddick, O., \& Atkinson, J. (2013). Visual control of manual actions: brain mechanisms in typical development and developmental disorders. Developmental Medicine and Child Neurology, 55(s4), 13-18. https://doi.org/10.1111/dmon.12300.

Broadbent, H. J., Farran, E. K., \& Tolmie, A. (2014). Egocentric and allocentric navigation strategies in Williams syndrome and typical development. Developmental Science, 17(6), 920-934. https://doi. org/10.1111/desc.12176.

Brouwer, S. I., van Beijsterveldt, T. C. E. M., Bartels, M., Hudziak, J. J., \& Boomsma, D. I. (2006). Influences on achieving motor milestones: a twin-singleton study. Twin Research and Human Genetics, 9(3), 424-430. https://doi.org/10.1375/twin.9.3.424.

Bruininks, H. (1978). Bruininks-Oseretsky test of motor proficiency. Circle Pines: American Guidance Service.

Bruininks, R. H., \& Bruininks, B. D. (2005). Bruininks-Oseretsky test of motor proficiency, (BOT-2). Minneapolis: Pearson Assessment.

Bushnell, E. W., \& Boudreau, J. P. (1993). Motor development and the mind: the potential role of motor abilities as a determinant of aspects of perceptual development. Child Development, 64(4), 1005-1021. https://doi.org/10.1111/j.1467-8624.1993.tb04184.x.

Carrasco, X., Castillo, S., Aravena, T., Rothhammer, P., \& Aboitiz, F. (2005). Williams syndrome: pediatric, neurologic, and cognitive development. Pediatric Neurology, 32(3), 166-172. https://doi. org/10.1016/j.pediatrneurol.2004.09.013.

Chapman, C. S., \& Goodale, M. A. (2008). Missing in action: the effect of obstacle position and size on avoidance while reaching. Experimental Brain Research, 191(1), 83-97. https://doi.org/10. 1007/s00221-008-1499-1.

Chen, M., \& Bargh, J. A. (1999). Consequences of automatic evaluation: immediate behavioral predispositions to approach or avoid the stimulus. Personality and Social Psychology Bulletin, 25(2), 215-224. https://doi.org/10.1177/0146167299025002007.

Chiang, M. C., Reiss, A. L., Lee, A. D., Bellugi, U., Galaburda, A. M., Korenberg, J. R., et al. (2007). 3D pattern of brain abnormalities in Williams syndrome visualized using tensor-based morphometry. Neuroimage, 36(4), 1096-1109. https://doi.org/10.1016/j. neuroimage.2007.04.024.

Clearfield, M. W. (2004). The role of crawling and walking experience in infant spatial memory. Journal of Experimental Child Psychology, 89(3), 214-241. https://doi.org/10.1016/j.jecp.2004.07.003.

Coombes, S. A., Janelle, C. M., \& Duley, A. R. (2005). Emotion and motor control: movement attributes following affective picture processing. Journal of Motor Behavior, 37(6), 425-436. https://doi.org/ 10.3200/JMBR.37.6.425-436.

Coombes, S. A., Cauraugh, J. H., \& Janelle, C. M. (2006). Emotion and movement: activation of defensive circuitry alters the magnitude of a sustained muscle contraction. Neuroscience Letters, 396(3), 192 196. https://doi.org/10.1016/j.neulet.2005.11.048.

Coombes, S. A., Gamble, K. M., Cauraugh, J. H., \& Janelle, C. M. (2008). Emotional states alter force control during a feedback occluded motor task. Emotion, 8(1), 104. https://doi.org/10.1037/ 1528-3542.8.1.104

Cowie, D., Braddick, O., \& Atkinson, J. (2012). Visually guided step descent in children with Williams syndrome. Developmental
Science, 15(1), 74-86. https://doi.org/10.1111/j.1467-7687.2011. 01104.x.

Cummins, A., Piek, J. P., \& Dyck, M. J. (2005). Motor coordination, empathy, and social behaviour in school-aged children. Developmental Medicine and Child Neurology, 47(7), 437-442. https://doi.org/10.1017/S001216220500085X.

Deitz, J. C., Kartin, D., \& Kopp, K. (2007). Review of the BruininksOseretsky test of motor proficiency, (BOT-2). Physical \& Occupational Therapy in Pediatrics, 27(4), 87-102. https://doi. org/10.1080/J006v27n04 06.

Dilts, C. V., Morris, C. A., \& Leonard, C. O. (1990). Hypothesis for development of a behavioral phenotype in Williams syndrome. American Journal of Medical Genetics, 37(S6), 126-131. https:// doi.org/10.1002/ajmg.1320370622.

Dunn, L. M., Dunn, D. M., Styles, B., \& Sewell, J. (2009). The British picture vocabulary scale (3rd ed.). London: GL Assessment.

Elliott, C. D., Smith, P., \& McCulloch, K. (1996). British Ability Scales second edition (BAS II): administration and scoring manual. London: NFER-Nelson.

Farran, E. K., \& Jarrold, C. (2003). Visuospatial cognition in Williams syndrome: reviewing and accounting for the strengths and weaknesses in performance. Developmental Neuropsychology, 23(1-2), 173-200. https://doi.org/10.1080/87565641.2003.9651891.

Farran, E. K., Jarrold, C., \& Gathercole, S. E. (2001). Block design performance in the Williams syndrome phenotype: a problem with mental imagery? Journal of Child Psychology and Psychiatry, 42(06), 719-728. https://doi.org/10.1017/S0021963001007429.

Farran, E. K., Bowler, A., Karmiloff-Smith, A., D’Souza, H., Mayall, L., \& Hill, E. L. (2019). Cross-domain associations between motor ability, independent exploration and large-scale spatial navigation; Attention Deficit Hyperactivity Disorder, Williams syndrome and typical development. Frontiers in Human Neuroscience, 13, 225. https://doi.org/10.3389/fnhum.2019.00225.

Farran, E.K., Bowler, A., Hill, E.L., D'Souza, H., Mayall, L., Brady, D., Sumner, E., Karmiloff-Smith, A. (2020). Is the motor impairment in Attention Deficit Hyperactivity Disorder (ADHD) a co-occurring deficit or a phenotypic characteristic? Advances in Neurodevelopmental Disorders. Advance of Print. https://doi.org/ 10.1007/s41252-020-00159-6.

Frick, A., \& Möhring, W. (2013). Mental object rotation and motor development in 8-and 10-month-old infants. Journal of Experimental Child Psychology, 115(4), 708-720. https://doi.org/10.1016/j.jecp. 2013.04.001.

Gosch, A., \& Pankau, R. (1994). Social-emotional and behavioral adjustment in children with Williams-Beuren syndrome. American Journal of Medical Genetics, 53(4), 335-339. https://doi.org/10. 1002/ajmg.1320530406.

Green, D., Baird, G., \& Sugden, D. (2006). A pilot study of psychopathology in developmental coordination disorder. Child: Care, Health and Development, 32(6), 741. https://doi.org/10.1111/j. 1365-2214.2006.00684.x.

Greer, M. K., Brown III, F. R., Pai, G. S., Choudry, S. H., \& Klein, A. J. (1997). Cognitive, adaptive, and behavioral characteristics of Williams syndrome. American Journal of Medical Genetics, 74(5), 521-525. https://doi.org/10.1002/(SICI)1096-8628(19970919)74: $5<521::$ AID-AJMG13>3.0.CO;2-E.

Gross, M. M., Crane, E. A., \& Fredrickson, B. L. (2012). Effort-shape and kinematic assessment of bodily expression of emotion during gait. Human Movement Science, 31(1), 202-221. https://doi.org/10. 1016/j.humov.2011.05.001.

Harter, S. (1987) The determinants and mediational role of global selfworth in children. In N. Eisenberg (Ed.), Developmental psychology (pp. 219-242). New York: John Wiley, New York.

Henderson, S. E., Sugden, D. A., \& Barnett, A. L. (2007). Movement assessment battery for children-second edition. London, UK: The Psychological Corporation. 
Hill, E. L., \& Brown, D. (2013). Mood impairments in adults previously diagnosed with developmental coordination disorder. Journal of Mental Health, 22(4), 334-340. https://doi.org/10.3109/09638237. 2012.745187

Hocking, D. R., Bradshaw, J. L., \& Rinehart, N. J. (2008). Fronto-parietal and cerebellar contributions to motor dysfunction in Williams syndrome: a review and future directions. Neuroscience \& Biobehavioral Reviews, 32(3), 497-507. https://doi.org/10.1016/j. neubiorev.2007.09.003.

Hocking, D. R., Rinehart, N. J., McGinley, J. L., Galna, B., Moss, S. A., \& Bradshaw, J. L. (2011). Gait adaptation during obstacle crossing reveals impairments in the visual control of locomotion in Williams syndrome. Neuroscience, 197, 320-329. https://doi.org/10.1016/j. jns.2010.11.001.

Iverson, J. M. (2010). Developing language in a developing body: the relationship between motor development and language development. Journal of Child Language, 37(2), 229-261. https://doi.org/ 10.1017/S0305000909990432.

Jax, S. A., \& Rosenbaum, D. A. (2009). Hand path priming in manual obstacle avoidance: rapid decay of dorsal stream information. Neuropsychologia, 47(6), 1573-1577. https://doi.org/10.1016/j. neuropsychologia.2008.05.019.

Jernigan, T. L., \& Bellugi, U. (1990). Anomalous brain morphology on magnetic resonance images in Williams syndrome and Down syndrome. Archives of Neurology, 47(5), 529-533. https://doi.org/10. 1001/archneur.1990.00530050049011.

Jernigan, T. L., Bellugi, U., Sowell, E., Doherty, S., \& Hesselink, J. R. (1993). Cerebral morphologic distinctions between Williams and Down syndromes. Archives of Neurology, 50(2), 186-191. https:// doi.org/10.1001/archneur.1993.00540020062019.

Jones, W., Hesselink, J., Duncan, T., Matsuda, K., \& Bellugi, U. (2002). Cerebellar abnormalities in infants and toddlers with Williams syndrome. Developmental Medicine and Child Neurology, 44(10), 688-694. https://doi.org/10.1111/j.1469-8749.2002.tb00271.xMABC.

Kamirloff-Smith, A. (1998). Development itself is the key to the understanding developmental disorders. Trends in Cognitive Sciences, 2, 389-398. https://doi.org/10.1016/S1364-6613(98)01230-3.

Lenhoff, H. M., Wang, P. P., Greenberg, F., \& Bellugi, U. (1997). Williams syndrome and the brain. Scientific American, 277(6), 68-73 Retrieved from: http://www.jstor.org/stable/24996044.

Martin, N. D., Snodgrass, G. J., \& Cohen, R. D. (1984). Idiopathic infantile hypercalcaemia-a continuing enigma. Archives of Disease in Childhood, 59(7), 605-613 https://doi.org/10.1136/adc.59.7.605.

Mervis, C. B., Klein-Tasman, B. P., \& Mastin, M. E. (2001). Adaptive behavior of 4-through 8-year-old children with Williams syndrome. American Journal on Mental Retardation, 106(1), 82-93 Retrieved from: http://www.aaiddjournals.org.libproxy.ucl.ac.uk/doi/abs/10. 1352/0895-8017(2001)106\%3C0082:ABOTYO\%3E2.0.CO;2? code $=$ aamr-site

Monchi, O., Petrides, M., Strafella, A. P., Worsley, K. J., \& Doyon, J. (2006). Functional role of the basal ganglia in the planning and execution of actions. Annals of Neurology: Official Journal of the American Neurological Association and the Child Neurology Society, 59(2), 257-264. https://doi.org/10.1002/ana.20742.

Morris, C. A., \& Mervis, C. B. (1999). Williams syndrome. In S. Goldstein \& C. R. Reynolds (Eds.), Handbook of neurodevelopmental and genetic disorders in children (pp. 555590). New York: The Guilford Press.

Nordstrøm, M., Hansen, B. H., Paus, B., \& Kolset, S. O. (2013). Accelerometer-determined physical activity and walking capacity in persons with Down syndrome, Williams syndrome and PraderWilli syndrome. Research in Developmental Disabilities, 34(12), 4395-4403. https://doi.org/10.1016/j.ridd.2013.09.021.

Osório, A., Soares, J. M., Prieto, M. F., Vasconcelos, C., Fernandes, C., Sousa, S., et al. (2014). Cerebral and cerebellar MRI volumes in
Williams syndrome. Research in Developmental Disabilities, 35(4), 922-928. https://doi.org/10.1016/j.ridd.2013.12.014.

Papaeliou, C., Polemikos, N., Fryssira, E., Kodakos, A., Kaila, M., Yiota, X., et al. (2012). Behavioural profile and maternal stress in Greek young children with Williams syndrome. Child: Care, Health and Development, 38(6), 844-853. https://doi.org/10.1111/j.1365-2214. 2011.01306.x.

Rasmussen, L. B., Andersen, L. F., Borodulin, K., Enghardt Barbieri, H., Fagt, S., Matthiessen, J., Sveinsson, T., Thorgeirsdottir, H., Trolle, E. (2012). Nordic monitoring of diet, physical activity and overweight: first collection of data in all Nordic countries 2011. Nordic Council of Ministers. https://doi.org/10.6027/TN2012-552.

Raven, J., Raven, J. A., \& Raven, J. C. (2003). Manual for Raven's progressive matrices and vocabulary scales: Section 1: general overview. Oxford, UK: Oxford Psychologists Press.

Reiss, A. L., Eliez, S., Schmitt, J. E., Straus, E., Lai, Z., Jones, W., \& Bellugi, U. (2000). IV. Neuroanatomy of Williams syndrome: a high-resolution MRI study. Journal of Cognitive Neuroscience, 12 (Supplement 1), 65-73. https://doi.org/10.1162/ 089892900561986.

Rotteveel, M., \& Phaf, R. H. (2004). Automatic affective evaluation does not automatically predispose for arm flexion and extension. Emotion, 4(2), 156. https://doi.org/10.1037/1528-3542.4.2.156.

Schindler, I., Rice, N. J., McIntosh, R. D., Rossetti, Y., Vighetto, A., \& Milner, A. D. (2004). Automatic avoidance of obstacles is a dorsal stream function: evidence from optic ataxia. Nature Neuroscience, 7(7), 779. https://doi.org/10.1038/nn1273.

Schneider, T., Skitt, Z., Liu, Y., Deacon, R. M., Flint, J., KarmiloffSmith, A., et al. (2012). Anxious, hypoactive phenotype combined with motor deficits in Gtf2ird1 null mouse model relevant to Williams syndrome. Behavioural Brain Research, 233(2), 458473. https://doi.org/10.1016/j.bbr.2012.05.014.

Schoemaker, M. M., \& Kalverboer, A. F. (1994). Social and affective problems of children who are clumsy: how early do they begin? Adapted Physical Activity Quarterly, 11(2), 130-140. https://doi. org/10.1123/apaq.11.2.130.

Schwarzer, G., Freitag, C., Buckel, R., \& Lofruthe, A. (2013). Crawling is associated with mental rotation ability by 9 -month-old infants. Infancy, 18(3), 432-441. https://doi.org/10.1111/j.1532-7078. 2012.00132.x.

Shaffer, D. D. R., \& Kipp, K. (2010). Developmental psychology: childhood \& adolescence: childhood and adolescence. Cengage Learning.

Skinner, R. A., \& Piek, J. P. (2001). Psychosocial implications of poor motor coordination in children and adolescents. Human Movement Science, 20(1-2), 73-94. https://doi.org/10.1016/S0167-9457(01) 00029-X.

Smits-Engelsman, B., \& Hill, E. L. (2012). The relationship between motor coordination and intelligence across the IQ range. Pediatrics, peds-2011. https://doi.org/10.1542/peds.2011-3712.

Stinton, C., Elison, S., \& Howlin, P. (2010). Mental health problems in adults with Williams syndrome. American Journal on Intellectual and Developmental Disabilities, 115(1), 3-18. https://doi.org/10. 1352/1944-7558-115.1.3.

Stodden, D. F., Goodway, J. D., Langendorfer, S. J., Roberton, M. A., Rudisill, M. E., Garcia, C., \& Garcia, L. E. (2008). A developmental perspective on the role of motor skill competence in physical activity: an emergent relationship. Quest, 60(2), 290-306 http://www. tandfonline.com/doi/abs/10.1080/00336297.2008.10483582.

Sumner, E., Leonard, H. C., \& Hill, E. L. (2016). Overlapping phenotypes in autism spectrum disorder and developmental coordination disorder: a cross-syndrome comparison of motor and social skills. Journal of Autism and Developmental Disorders, 46(8), 26092620. https://doi.org/10.1007/s10803-016-2794-5.

Thelen, E., \& Smith, L. B. (1998). Dynamic systems theories. In W. Damon \& R. M. Lerner (Eds.), Handbook of child psychology: 
theoretical models of human development (pp. 563-634). Hoboken: Wiley.

Tsai, S. W., Wu, S. K., Liou, Y. M., \& Shu, S. G. (2008). Early development in Williams syndrome. Pediatrics International, 50(2), 221-224. https://doi.org/10.1111/j.1442-200X.2008.02563.x.

Udwin, O. (1990). A survey of adults with Williams syndrome and idiopathic infantile hypercalcaemia. Developmental Medicine and Child Neurology, 32(2), 129-141. https://doi.org/10.1111/j.1469-8749. 1990.tb16912.x.

Ulrich, B. D. (1987). Perceptions of physical competence, motor competence, and participation in organized sport: their interrelationships in young children. Research Quarterly for Exercise and Sport, 58(1), 57-67. https://doi.org/10.1080/02701367.1987.10605421.

Wagner, M. O., Bös, K., Jascenoka, J., Jekauc, D., \& Petermann, F. (2012). Peer problems mediate the relationship between developmental coordination disorder and behavioral problems in schoolaged children. Research in Developmental Disabilities, 33(6), 2072. https://doi.org/10.1016/j.ridd.2012.05.012.

Wang, P. P., \& Bellugi, U. (1994). Evidence from two genetic syndromes for a dissociation between verbal and visual-spatial short-term memory. Journal of Clinical and Experimental Neuropsychology, 16(2), 317-322. https://doi.org/10.1080/01688639408402641.

Watkinson, E. J., Dunn, J. C., Cavaliere, N., Calzonetti, K., Wilhelm, L., \& Dwyer, S. (2001). Engagement in playground activities as a criterion for diagnosing developmental coordination disorder. Adapted Physical Activity Quarterly, 18(1), 18-34. https://doi.org/10.1123/ apaq.18.1.18.
Who Multicentre Growth Reference Study Group, \& de Onis, M. (2006). WHO Motor Development Study: windows of achievement for six gross motor development milestones. Acta Paediatrica, 95, 86-95. https://doi.org/10.1111/j.1651-2227.2006.tb02379.x.

Withers, S. (1996). A new clinical sign in Williams syndrome. Archives of Disease in Childhood, 75(1), 89. https://doi.org/10.1136/adc.75. 1.89 .

Woodruff-Borden, J., Kistler, D. J., Henderson, D. R., Crawford, N. A., \& Mervis, C. B. (2010). Longitudinal course of anxiety in children and adolescents with Williams syndrome. American Journal of Medical Genetics Part C: Seminars in Medical Genetics, 154(2), 277-290. https://doi.org/10.1002/ajmg.c.30259 Hoboken: Wiley Subscription Services, Inc., A Wiley Company.

Wrotniak, B. H., Epstein, L. H., Dorn, J. M., Jones, K. E., \& Kondilis, V. A. (2006). The relationship between motor proficiency and physical activity in children. Pediatrics, 118(6), e1758-e1765. https://doi. org/10.1542/peds.2006-0742.

Wuang, Y. P., \& Tsai, H. Y. (2017). Sensorimotor and visual perceptual functioning in school-aged children with Williams syndrome. Journal of Intellectual Disability Research, 61(4), 348-362. https://doi.org/10.1111/jir.12346.

Publisher's Note Springer Nature remains neutral with regard to jurisdictional claims in published maps and institutional affiliations. 